\title{
An RFID Based Technique for Handling Object Distribution and Recalls in Pervasive Transaction Environments
}

\author{
Pradip De, Kalyan Basu and Sajal K. Das \\ Center for Research in Wireless Mobility and Networking(CReWMaN) \\ Department of Computer Science and Engineering \\ University of Texas at Arlington, TX 76019-0015 \\ \{pradipde, basu, das\}@cse.uta.edu
}

\begin{abstract}
A completely visible Pervasive Transaction Environment where it is possible to link all related distributions of physical objects and trace their mobility through their entire life process, has been elusive. With the emergence of Radio Frequency Identification (RFID) based object tags, it is now practicable to automatically collect information pertaining to the object's place, time, transaction, etc. We propose an architecture for pervasive real-time tracking of object distribution and subsequently present an efficient mechanism for performing Recall of RFID tagged objects that were previously distributed but turned out defective. Applying the concepts of Scale Free Networks and Epidemic Theory, we mathematically model the distribution and recall process and get a stochastic estimate of the average spread of object distributions and the number of recall messages required. Our simulation results validate the analysis and the small average number of recall messages obtained proves the efficacy of the scheme.
\end{abstract}

\section{Keywords}

RFID, Distributed Transactions, Tracking, Object Recall, Epidemic Theory, Scale Free Networks.

\section{Introduction}

The management of distributed transactions [2] of physical objects among different organizations, and their real-time mobility tracking [3], [4], [8] offer major challenges. With the globalization of today's market and the increased complexity in the international supply demand relationship, the supply chain has been exposed to higher risks. Mismanagement, leading to excessive or mismatched inventory, contributes to the risk of reworking stock and penalties for non-delivery of goods and hence precipitates financial losses. Moreover, there is always the human error factor which adds to the woes of supply chain management.

Thus, an efficient framework for automatically tracking physical objects transacted between organizations is es- sential and would not only reap huge benefits in terms of saved time and loss avoidance, but also provide a pervasive environment for organizations to seamlessly and efficiently implement their transactions, when globally deployed. Moreover, the effects of efficient automatic object tracking would also help in an entirely different scenario which often manifests itself and has received little attention is the situation of an Object Recall. Substantial losses are incurred because no efficient technique is known by which an organization can recall all its (distributed) defective objects fast enough to avoid any associated damage. The problem lies in locating all the points of distribution and sending a recall message to those points within a very short time.

Radio Frequency Identification (RFID) technology [10], [9] has emerged as a practical solution to aid automatic object identification and tracking. These wireless systems enable non-contact reading from a distance and their deployment is highly effective in manufacturing and other hostile environments where the employment of bar code labels was infeasible. This technology can be used very efficiently to pervasively tag physical objects so that strategically placed RFID readers sensing them can track the mobile objects through the entire transaction processes. By the term transaction, we basically qualify the movement of a tagged object from its source to destination passing through different organizations and hierarchy and possibly changing ownership in the process.

Our Contributions: In this paper, we propose a new architecture RFArch that could be globally deployed for tracking inter-organizational transactions of tagged objects in real-time. This architecture being generic can be deployed in various scenarios. Its inherent distributed nature takes care of scalability issues and fits well with the implementation of different heterogeneous Process Level Agreements (PLA) between various organizations.

Currently there is no proper, efficient and standardized method by which organizations can actually implement an 
object recall. Based on the proposed architecture for location tracking of transacted physical objects, we propose distributed Recall Algorithms to be deployed at each node of the network to automatically handle the situation of a Recall.

Next, we theoretically analyze and get a stochastic estimate of the average spread of object distributions and the amount of messaging necessary for an Object Recall based on the concepts of Scale Free Networks [14] and Epidemic Theory [12]. We show how our distribution network is fundamentally scale free and then based on the dynamics of an infection spread we model our distribution and stochastically obtain the fraction of the total nodes in the network to which the distribution spreads.

Finally, we describe a discrete event driven simulation of a recall scenario in a network and estimate the number of messages per tag generated to perform the recall. Our results show that the number of messages is indeed very small and the use of this recall handling framework can provide high performance benefits.

The rest of the paper is organized as follows: Section II provides an overview of the network architecture that would be deployed by organizations for automating their distributed transaction processes. Section III outlines the Object Recall Process and the algorithms that would be deployed at each node of the distribution network. In Section IV we provide a mathematical model and analysis of the object distribution and recall process within the architecture based on the concepts of Epidemiology and Scale Free Networks. The simulation details, necessary assumptions and experimental results are presented in section $\mathrm{V}$ and finally, we conclude in section VI.

\section{The Tracking Architecture - RFArch}

In this section, we describe the distributed architecture, RFArch, that would be deployed globally and form the underlying framework for performing inter-organization transactions of tagged physical objects. Each tag would basically have an electronic product code (EPC) [5] associated with it. EPC has been standardized by the AutoID Center for uniquely identifying every manufactured physical object. The components of RFArch as depicted in figure 1 are (i) RFID Readers, (ii) Savants and (iii) PML Servers. RFArch is hierarchical in nature, organized as a balanced tree topology. At the leaves of this topology, multiple RFID readers are engaged in continually picking up EPC tag information attached to the mobile objects. For every EPC, the reader basically forwards the following information: (i)the EPC of the tag read, (ii) the EPC or ID of the reader that scanned the above tag, (iii) the reading timestamp and (iv) any non-EPC related information, such as temperature or geographical position observed by the reader.

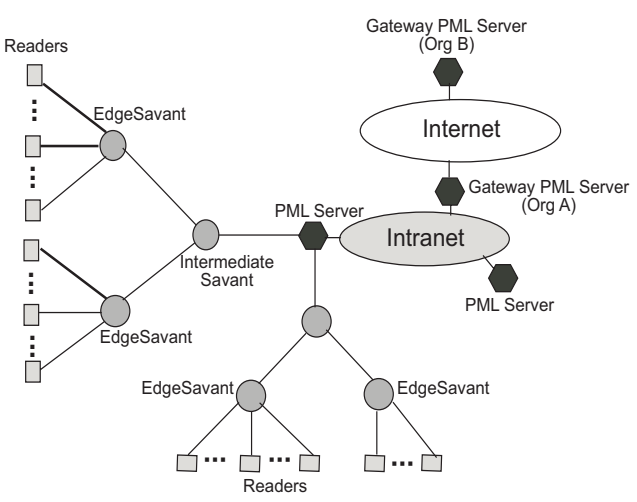

Fig. 1. The Architecture Schematic

Since the readers at the leaves consume a huge amount of data inflow coming from the plentitude of mobile objects that enter and leave their radio range, this location information needs to be continually updated. Several RFID readers are thus homed at a fast data routing server residing at the next higher level of the hierarchy called Savant [6]. The concept of Savant was also standardized at the MIT Auto-ID Center and we make use of them in RFArch. It is a data router that performs operations like capturing, monitoring and transmission of the input data coming from the RFID readers. The savants directly attached to the readers are called the Edge Savants. Readers of different workspace areas are connected to a Savant, based on predefined spatial and topological rules. There are Internal Savants that reside above the Edge Savants in the topology. They mainly aggregate the raw data collected by their descendants and deal with forwarding the aggregated data upwards. Details about how the Savant works are dealt with in [6]. In RFArch, these Savants would serve to forward location update information from the readers to another component of our architecture i.e., the PML server which resides at the next higher level of the topology. Based on the constructs of the popular eXtensible Markup Language (XML), the MIT-AutoID Center has come up with a new language for describing physical objects termed The Physical Markup Language(PML)[7]. All the complexity of describing and classifying objects have moved from the object label to the PML file which contains all the information pertaining to the object. The PML server is basically the storehouse where all object information (static and dynamic) are stored in PML files. Each site or domain of an organization would be controlled by a PML server. For instance, we can envision each WalMart outlet to be having a Savant Framework and their corresponding readers and a collection of a few outlets in a small town to be controlled by a single PML Server. Every organization would have at least one PML server which 
stores all the information of objects that that organization owns. This PML Server is called the Home PML Server $(H-P M L)$ and is supposed to own that object. Analogously, in an airport scenario, where all tickets would be tagged, the H-PML of a tagged ticket would be a PML Server of the airlines company located at the city of departure.

The PML servers of an organization would be internetworked by the organization's intranet as depicted in Fig 1. The dynamic data corresponding to an object is basically serial level tracking and location centric mobility information and would be stored in mobility management databases. Following on similar lines as the Mobile Cellular Systems, we propose that every EPC would be associated with its Home Location Database or HLD that will keep all its location information. Whenever, there is a change in location of a particular EPC, the corresponding PML server would notify the H-PML which would update the associated HLD. The HLD also keeps a history of the PML servers visited by the EPC and the corresponding timestamps. Likewise, a dynamically changing Temporary Location Database or TLD cache would be located at corresponding PML Servers to keep temporary information about the object's location which has currently entered its domain. When a new object enters the field of a particular RFID reader, the reader relays the location information to the corresponding PML Server. This PML Server, after updating the EPC's H-PML about the object's new location, copies all relevant information for this EPC from its H-PML into its TLD. By relevant information it is implicitly assumed that information is exchanged depending on the organizations' respective Process Level Agreements (PLAs). Thus, in a typical organization's network, there would be a HLD and TLD associated with each PML Server that would take care of location information of the organization's EPCs. In addition to storing objects' information, PML servers are configured to answer queries coming from other organizations for information on any particular EPC. These queries are answered by providing the respective PML file fragment containing the required information for the specific EPC. To handle queries and transactions with other organizations, one of the PML Servers would perform Gateway Functions to facilitate inter-organizational transaction processes. We call this server the Gateway PML Server (GW-PML).

Two transacting organizations have their independent RFID networks that would interwork via their respective GW-PMLs. The GW-PMLs would possibly act as protocol converters between the two implementations so that the transaction process will extend its scope beyond its own area. The protocol conversion will include both Semantic and Syntactic conversion mechanisms that are defined apriori based on the PLAs of the two organizations. This conversion of the messages between the two systems based on their PLA will allow any two systems to interwork seamlessly once an PLA has been setup between them.

Based on the above architecture, organizations would use a tracking mechanism by exchanging location update information whenever objects are transacted between them and update their respective HLD and TLD accordingly. We have devised a tracking protocol based on this architecture which is detailed in [1] and could not be incorporated due to space constraints.

\section{Object Recall}

In this section we talk about a post-distribution scenario which has not received much attention till date although it is of prime importance. After the objects have been distributed down the Supply Chain hierarchy to the destination they can possibly be forgotten. This poses a potential problem in the scenario when certain objects that were distributed turn out to be defective. It becomes important and mandatory for the manufacturing organization to be able to recall back those defective objects which have percolated down the Supply Chain before substantial damage is done. Our tracking architecture comes in handy in such a situation. The location history that is stored at the $H L D$ gives the entire path that that EPC traversed since its inception. It is based on this notion that our Recall works Traverse the footsteps of the EPC distribution phase. Since the EPCs need to be brought back to the manufacturing organization, it is obvious that a recall starts at the H-PML which is the owner of the EPCs under question. Let the set of EPCs to be recalled be denoted by $E$. The primary task before a recall can be initiated is the building of a Distribution Location Trie which we shall call the Recall Trie $T$. This trie is built from the HLD using the location history information. Each node $i$ of the trie corresponds to a PML server and contains a subset $E^{i}$ of $E$ denoting the set of EPCs that have travelled from the root to $i$. In other words, $T$ emulates the distribution phase of the Recall $E P C s$ with the root of $T$ containing the full set of EPCs $E$. This set $E$ gets distributed in the recall trie $T$ in the manner the EPCs were actually delivered to corresponding PML servers when the transactions took place.

Our Recall Scenario consists of two contexts. The first context is with respect to an initiation of a Recall which happens at the H-PML and the second context pertains to handling a recall arrival which takes place at every PML Server that a recall traverses through. Based on these two contexts we propose two algorithms running at every PML Server for handling recall in the network: Recall Initiation Algorithm, and Recall Handling Algorithm. They are described below.

To start a recall process, the H-PML would invoke the Recall Initiation procedure to calculate the set of PML servers to which the recall would ultimately be sent. It uses 
the EPC set $E$ to be recalled and the information stored in $H L D$ to identify the distinct PML servers where different subsets of $E$ have been sent by constructing a Recall Trie $T$ in the process. This process would then spawn recalls corresponding to each PML server where a subset of $E$ has been distributed.

These PML servers, on receiving the recalls would invoke the Process_Recall procedure and raise a recall interrupt within their domain. As an optional function, the Process_Recall procedure, after completion of all the critical tasks pertaining to recall handling, would also notify its immediate predecessor PML server (during distribution) of the Recall.

The string of PML servers that an EPC traverses to, may consist of one or more distributions. Every two consecutive distribution represents a mapping of an EPC from the first distribution to an EPC of the second. This happens when an encapsulation/decapsulation or an assembly/disassembly occurs. Mapping can also mark a change of ownership of the object. During a recall scenario, there is one recall message generated for each distribution in the chain from the root of each to the destination PML server. If there were a subsequent distribution from this PML server, then a new recall message would be transmitted to the end node of this distribution and so on.

Within the Process_Recall procedure, these PML servers would then map the arrived EPC set onto another EPC set and initiate a new recall by invoking their respective Initiate_Recall procedures with the new generated EPC set. Let us formally define the recall as follows.

Let $P_{t}$ denote the set of PML servers of organization $t$. Therefore,

$P_{t}=\left\{P_{t}^{1}, \ldots, P_{t}^{n}\right\}$ where $P_{t}^{i}$ is the $i^{t h}$ PML Server of organization $t$.

Let us assume that $P_{t}^{i}$ initiates a recall $R_{t}^{i}$ which is defined as

$R_{t}^{i}=\left\{E_{t}^{i}, C_{t}^{i}, P F_{t}^{i}\right\}$ where

$E_{t}^{i}$ is the EPC set being recalled.

$C_{t}^{i}$ is the list of PML server nodes from the Recall Tree $T$ such that the EPC set $E_{t}^{i}$ traversed all the nodes of $C_{t}^{i}$.

$P F_{t}^{i}$ is the fragment of the PML file containing information about the EPC set $E_{t}^{i}$.

If the recall propagates further, then the current PML server performs a Mapping Function $\Psi$ on the current set of EPCs $E$ which results in a new set $E_{n e w}$. If a mapping takes place, then a new recall trie is constructed with the new mapped EPC set using the PML file fragment which came with the old recall and its current $H L D$. This PML server then becomes the H-PML for this new EPC set and the recall process is repeated. Thus,

$E_{\text {new }}=\Psi(E)$ and the new Recall is

$R_{\text {new }}=\left\{E_{\text {new }}, C_{\text {new }}, P F_{\text {new }}\right\}$

\section{A. The Initiate_Recall Algorithm}

This Algorithm starts at the H-PML and takes as input the set $E$ of EPCs to be recalled. $E$ is searched in $H L D$ and the location history list $C$ extracted, which contains the history of PML servers' location and timestamp for each EPC of the set. A subroutine BUILD_RECALL_TRIE is then invoked which takes each EPC's $C$ and incrementally constructs a trie for the whole EPC set $E$ recalled. Each node of the trie would represent a PML server $P$ with an associated EPC subset that traversed the chain of nodes from the root to that PML server during a distribution phase, earlier. In BUILD_RECALL_TRIE, we try to identify all the PML servers to which the set $E$ got distributed. We perform a Longest Prefix Match of $C$ with every spine of the trie and ultimately merge it with the spine having the longest intersection with $C$. The corresponding EPC sets of the end nodes of the spine get merged too.

After the trie is constructed, each node would have the EPC subset that traversed from the root to itself. They would then start sending recall messages to all the nodes which have a non empty set of EPCs associated with them. Note that all the EPCs in the set associated with a particular node have the same history chain denoted by the path from that node to the root of the trie. Optionally, this chain is also passed alongwith the recall so that the node can notify the previous PML servers in the chain about the incidence of the recall.

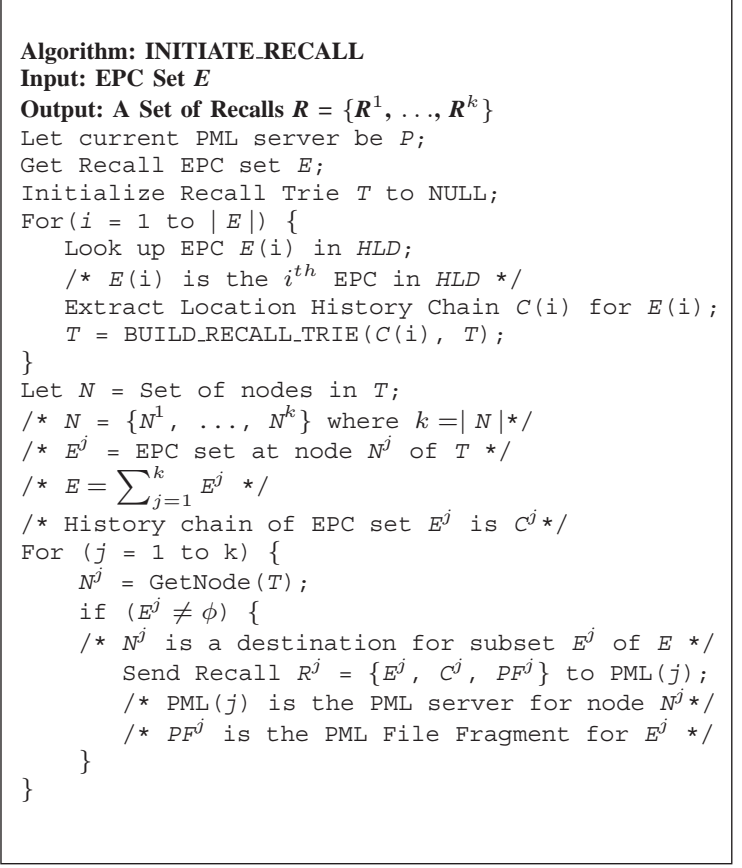

Fig. 2. Procedure of InitiateObjectRecall 


\section{B. Process_Recall Algorithm}

This algorithm would be invoked in all those PML servers that are the recipient of a recall from the H-PML of the current EPC set. It checks whether the EPC set was distributed further or not. If not, it generates the required Recall Alert and optionally, sends recall notifications to the history nodes in the arrived chain by stripping its entry off the chain $C$ and sending an acknowledgement containing $C$ to the next PML server in $C$ which processes it accordingly. Otherwise, the PML server performs the mapping and initiates a new recall corresponding to the new distribution. It may also notify the root of the current distribution about the propagation of the recall.

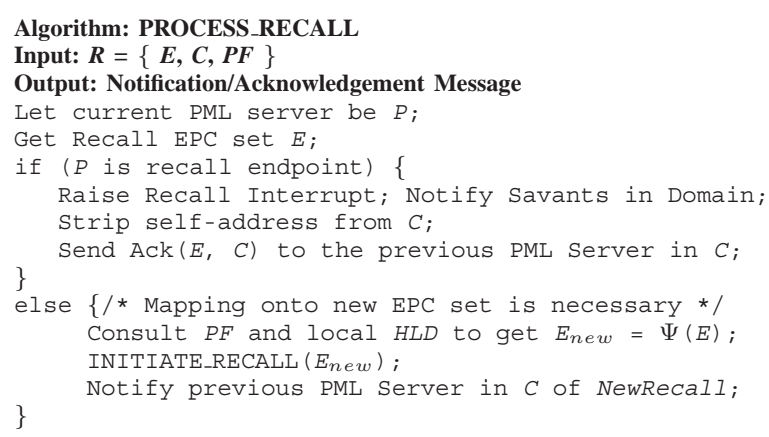

Fig. 3. Procedure of ProcessObjectRecall

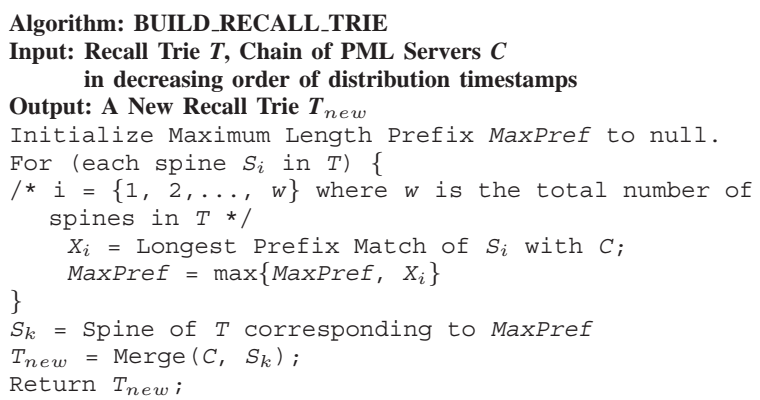

\section{Fig. 4. Procedure of BuildRecallTrie}

\section{EPC Distribution Modelling and Analysis}

In this section we stochastically analyze the distribution of the EPCs (every object being tagged by an EPC) among different organizations. Our analysis is based on modeling the Process Level Agreement (PLA) network among different PML servers of different organizations as a scale free topology. We show by analogy that the connectivity probability in the PLA graph which is a virtual topology over the physical PML server network underneath, follows the same principles as in Scale Free Networks [14]. On top of this scale free topology of the PLA network, we formulate the spreading of the EPC distribution by mathematically modeling it in a manner similar to the way an infection is transmitted in a population. Epidemic Theory throws much light on transmission dynamics of an infection [12].

Scale Free Networks: Scale-free networks[14] are topologies that are uniquely characterized by an unbalanced connectivity distribution. The nodes of these networks have a random pattern of connections, with some nodes having very high degree than others. These "highly connected" nodes form the hubs of the network and dramatically influences the way the network operates. The ratio of highly connected nodes to the number of nodes in the rest of the network remains constant as the network changes in size justifying the name "scale free". Previously, networks with complex topologies were described using random graph theory which basically states that there would be no well-connected nodes but the findings of [14] indicated the existence of a high degree of self organization characterizing the large-scale properties of complex networks. Exploring several large databases describing the topology of large networks that extend to fields as diverse as the WWW or citation patterns, it was shown that independent of the system and the identity of its constituents, the probability $P(\kappa)$ that a vertex in the network interacts with $\kappa$ other vertices decays as a power law, following $P(\kappa) \sim \kappa^{-\gamma}$. This result indicates that large networks self-organize into a scale-free state, a feature unpredicted by all existing random network models. To establish this assertion, it was proved by Barabasi and Albert [14] that existing network models fail to incorporate growth and preferential attachment, two key features of real networks.

The PLA network between different PML servers of various organizations can be thought to be a graph $G(V$, $E$ ) where each vertex $v \in V$ represents a distribution point and an edge $e=\left\{v_{1}, v_{2}\right\} \in E$ represents an PLA agreement between $v_{1}$ and $v_{2}$. For our analysis, we would consider each distribution point in the graph to represent an actual distribution site which is under the purview of a single PML Server. The end points of an edge in $G$ denote the two PML servers such that an PLA exists between them and their is a potential for an EPC distribution.

$G$ has two fundamental properties which is characteristic of Scale Free Networks: (i) It expands continuously as more organizations sign process level agreements, and (ii) The PLAs can be thought to be preferential to nodes that have a high degree which is indicative of the trust- 
worthiness of that node as regards to PLAs signed with it. $G$ basically gives a picture of the business relationships that different organizations have amongst each other and as a consequence of these two features, we observe that the vertex connectivities of $G$ follow a scale-free powerlaw distribution characteristic of Scale Free Networks.

We model the EPC distribution on top of the PLA network $G$ based on concepts from Epidemic Theory and how an infection released into a population, actually spreads.

Epidemiology: A key feature of epidemiology [12] is the measurement of infection outcomes in relation to a population at risk. The population at risk basically comprises the set of people who possess a susceptibility factor with respect to the infection. This factor is dependent on several parameters like exposure, spreading rate, previous frequency of occurrence etc. which define the potency of the disease causing the infection. Various models exist in epidemic theory which characterize an infection spread, such as Susceptible-Infected-Susceptible (S-I-S) Model, Susceptible-Infected-Recovered (S-I-R) Model etc. In the former, a susceptible individual acquires infection and then after an incubation period, (i.e., the time the infection persists), the individual becomes susceptible again. On the other hand, in the latter, the individual recovers and becomes immune to further infections.

An approach to model the spread of an infection is to assume that the probability (per unit time) for a susceptible individual to acquire infection is equal to the average rate at which new partners are acquired multiplied by the probability of being infected by any one partner.

In a basic model, where $N(t), X(t)$ and $Y(t)$ denote the total population, the susceptibles and infected individuals respectively at time t, we can say

$$
N(t)=X(t)+Y(t)
$$

If $D$ represents the average period of the infection, then $\frac{1}{D}$ denotes the average rate at which an individual changes from Infected to either Recovered or Susceptible state again. Other important parameters considered include the average death rate $\mu$ of an individual, the rate $B(t)$ at which new susceptibles enter into the population etc. The following first-order differential equations describe the dynamics of the population in the face of an infection[11].

$$
\begin{aligned}
\frac{d X(t)}{d t} & =B(t)-(c \lambda+\mu) X(t) \\
\frac{d Y(t)}{d t} & =c \lambda X(t)-\left(\frac{1}{D}+\mu\right) Y(t) \\
\frac{d N(t)}{d t} & =B(t)-\mu N(t)-\frac{1}{D} Y(t)
\end{aligned}
$$

Here $\mathrm{c} \lambda$ is the 'force of infection', representing the probability per unit time that a given susceptible would become infected where $\mathrm{c}$ is the average rate at which contacts happen between an infected and a susceptible and $\lambda$ is the probability of acquiring an infection from a contact with an individual. The fraction $I(t)=\frac{Y(t)}{N(t)}$ of individuals infected at time $t$ can be obtained by direct integration of the above equations. The steady state solution is obtained by considering the value of this fraction asymptotically in time.

Our Model and Analysis: In our EPC distribution model, the individuals are the PML servers and a distribution of EPCs from node $v_{1}$ to $v_{2}$ is analogous to a spread of infection from $v_{1}$ to $v_{2}$. In our model, we assume that initially the whole population of PML servers is susceptible to an incident distribution, i.e., $B$ in Eqn. 2 and 4 is zero. Moreover, $\mu=0$ because we assume that there is no removal of PML servers from the network once it is formed. For EPC distribution we use the S-I-S model for infection spreading. After acquiring infection, $v_{2}$ then redistributes the objects further to other nodes thereby infecting them and also loses its own infection in the process. However, contrary to the S-I-R model of epidemiology in which the individual recovers after infection, here the node becomes susceptible again to future distributions with a higher probability. We increase the probability to assert that a node having a fair history of distributions is more prone to receive future ones. From a realistic point of view, let us imagine a new organization signing up a process level agreement with another organization. The PLA is still not permanent but after several successful transactions, the mutual trust builds and the preference is clear.

We assume that the rate at which nodes again redistribute the arrived EPCs is $\frac{1}{D}$, where the average duration during which a node processes a distribution in order to redistribute it is $D$. In case of a recall propagation, $D$ is equal to the running time of the Recall Algorithm. This is analogous to the average period of the infection. Thus $\frac{1}{D}$ is the average rate at which a node loses its infection to become susceptible again.

Susceptibility of a particular node $i$ to acquire distribution from another node depends on two factors: (i) The total number of EPCs $N$ arriving for distribution currently and (ii) the count on the number of previous distribution of the same type through node $i$.

For a node $i$ we define $r$ as the average rate of contact of node $i$ with a potential EPC distributor. $r$ is given by

$$
r=\frac{\bar{\kappa}}{\text { Average time between transactions }}
$$

where $\bar{\kappa}$ is the average degree of a node in the virtual PLA network $G$. We shall denote the average value of a quantity Q by the notation $\bar{Q}$.

Let $\xi=\left[\xi_{1}, \xi_{2}, \ldots, \xi_{m}\right]$ be the probability vector of node $i$ such that $\xi_{j}$ is the probability of receiving EPCs of type $j$ (among possibly $m$ types) for distribution from 
any other distributor after it has been introduced into the network. This is basically the probability of node $i$ acquiring infection from any one infected node. In order to calculate these probabilities of acquiring an infection, each node $i$ would maintain a vector $h=\left[h_{1}, h_{2}, \ldots, h_{n}\right]$ where $h_{j} \forall j=1, \ldots, m$, represents the count of the number of distributions of type $j$ through node $i$. The total count $H=$ $\sum_{j=1}^{m} h_{j}$ and $h_{\max }=\max _{j=1}^{m}\left(h_{j}\right)$. For each distribution of type $j$ let

$$
M_{j}=\alpha\left(h_{j}\right)^{2}+(1-\alpha) N
$$

where $\mathrm{N}$ is the total number of EPCs currently distributed and $\alpha$ is the weighing factor associated with each parameter. We square $h_{j}$ to render it comparable to $N$. Then,

$$
\xi_{j}=M_{j} / M_{\max }
$$

such that

$$
M_{\max }=\alpha\left(h_{\max }\right)^{2}+(1-\alpha) N_{\max }
$$

In our analysis, we would be concerned with distribution of one particular type of EPC at a time and so the subscript ' $j$ ' can be disregarded. Thus $\xi$ would mean the probability for the current type of EPC being distributed. Now, $\frac{Y}{N}$ is the probability that any randomly selected PML server is infected by the current EPC distribution. If $\lambda$ is the probability of acquiring infection from any infected node then,

$$
\lambda=\xi \frac{Y(t)}{N(t)}
$$

After a node $i$ acquires a set of EPCs for distribution, it needs to redistribute to other nodes based on the same principles of infection spread dynamics. Let $S$ denote the time for node $i$ to process an incident distribution and pass it on to other neighboring nodes. Let $p$ be the probability that the distribution ends at node $i$. Therefore, $(1-p)$ denotes the probability that the node would pass on the infection to other nodes. Therefore, the expected processing time is given by

$$
E[S]=p \times E\left[S_{1}\right]+(1-p) \times E\left[S_{2}\right]
$$

represents the average incubation period of the distribution at a node where $\mathrm{E}\left[S_{1}\right]$ is the expected time to process distributions destined for node $i$ and $\mathrm{E}\left[S_{2}\right]$ is the expected time to process distributions which need to be propagated. Thus $\omega=1 / \mathrm{E}[\mathrm{S}]$ would denote the average rate of change of a node from infected to susceptible state again.

Let $\Re_{j}$ denote the average rate at which node $i$ would acquire EPCs of a particular type for distribution, i.e., an infection. Then

$$
\Re_{j}=r \times \lambda
$$

Let us define

$$
\wp=\xi \omega \bar{\kappa}
$$

as the average number of secondary nodes which receive EPCs after the introduction of a distribution in a single node of the network where we have assumed every node to be susceptible. The quantity $\bar{\kappa}$ is the average number of distribution neighbors a node has. A node $i$ is said to be the distribution neighbor of another node $j$ if there exists an PLA between them. The graph resulting from these distributions is our virtual distribution graph $G$ which is a scale free network as explained earlier. Note that $\wp$ is analogous to the average number of secondary infections in Epidemiology and is known as the basic reproductive number [12]. Rewriting the differential equations for our EPC distribution we get

$$
\begin{aligned}
& \frac{d X(t)}{d t}=-r \lambda X(t) \\
& \frac{d Y(t)}{d t}=r \lambda X(t)-\omega Y(t)
\end{aligned}
$$

From the above two equations we have

$$
\frac{d N(t)}{d t}=-\omega Y(t)
$$

From the deductions in epidemiology [11], [12] and direct integration of the above equations it can be shown that asymptotically, the fraction of nodes of $G$ to which the current EPC distribution crawls to can be expressed as

$$
F=\overline{1-e^{-\kappa \sigma}}
$$

where $\sigma$ is denoted by the equation

$$
\sigma=\wp\left[\frac{\overline{\kappa\left(1-e^{-\kappa \sigma}\right)}}{\bar{\kappa}^{2}}\right]
$$

The averages in the above equation can be calculated if we approximate the number of neighbors of the nodes of the network to be a continuous value. Based on our assumption that $G$ represents a scale free network with the probability of node degrees following a power law we let $\delta$ denote the minimum degree of a node. With $\kappa$ denoting the node degree, we thus have $P(\kappa)$ to be

$$
P(\kappa)=\frac{2 \delta^{2}}{\kappa^{3}}
$$

from the total probability equation

$$
\int_{\delta}^{\infty} P(\kappa) d \kappa=1
$$

Therefore, the average degree is given by

$$
\bar{\kappa}=\int_{\delta}^{\infty} \kappa P(\kappa) d \kappa=2 \delta
$$


Substitution of $\bar{\kappa}$ from Eqn. 20 into Eqn. 17 we get

$$
\sigma=\wp\left(\frac{2 \delta^{2}}{4 \delta^{2}}\right) \int_{\delta}^{\infty} \frac{\left(1-e^{-\kappa \sigma}\right)}{\kappa^{2}} d \kappa
$$

By substituting $\eta=\sigma \delta$ and $\kappa=t \delta$ we obtain

$$
\eta=\frac{\wp}{2} \int_{1}^{\infty} \frac{\left(1-e^{-\eta t}\right)}{t^{2}} d t=\frac{\wp}{2}\left[1-E_{2}(\eta)\right]
$$

where $E_{2}(\eta)$ denotes the exponential integral of the second kind of $\eta$.

Using the recurrence relation

$$
E_{n+1}(z)=\frac{1}{n}\left\{e^{-z}-z E_{n}(z)\right\}
$$

from [13], we simplify eqn 22 to obtain

$$
\eta=\frac{\left\{1-e^{-\eta}\right\}}{\frac{2}{\wp}-E_{1}(\eta)}
$$

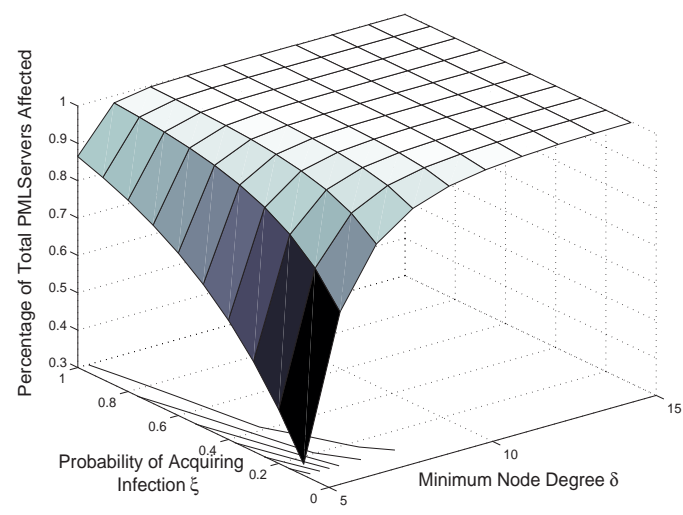

Fig. 5. Plot of Percentage of total PML servers infected

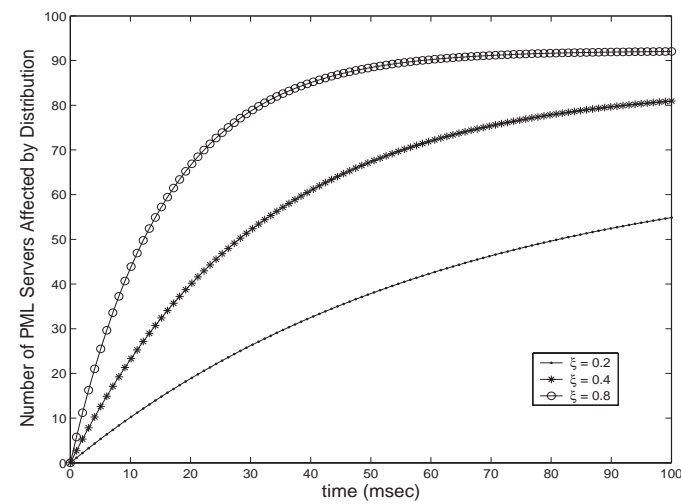

Fig. 6. Dynamics of distribution spread with time
Now $F$ is given by

$$
F=2 \delta^{2} \int_{\delta}^{\infty} \frac{\left(1-e^{-\kappa \sigma}\right)}{\kappa^{3}} d \kappa
$$

Having obtained $\eta(\wp)$ and using a similar substitution in Eqn 25 as previously done in Eqn 22 we get

$$
F=1-E_{3}(\eta)
$$

Expressing Eqn 26 in terms of $E_{1}(\eta)$ using the recurrence relation of Eqn 23 we get

$$
F=1-(1-\eta) e^{-\eta}-\eta^{2} E_{1}(\eta)
$$

In Figure 5, we show the percentage of PML servers which have received the distribution with respect to the probability of infection $\xi$ and the minimum degree of a node $\delta$. Figure 6 illustrates the dynamics of the count of the affected PML servers with time. The total number of PML servers is taken as 100. The three curves show the value of $Y(t)$ for three different values of infection probabilities.

\section{Simulation and Results}

This section outlines the simulation experiment for performing Object Recalls of distributed EPCs on a network of PML servers. In our experiment, we simulate how a Recall Algorithm running at each node the recall percolates to, would handle it. Our focus was primarily on how many messages were involved in a single recall. For this we average over a large number of recalls to get a proper estimate. We ignore the probability distribution of the recall inter-arrival times as we are only dealing with the number of messages involved.

Our simulation framework is based on an object oriented discrete event driven model implemented in $\mathrm{C}++$. The framework simulates two phases: (a)The EPC Distribution phase and (b)The EPC Recall phase

\section{A. EPC Distribution Phase}

In the distribution phase, an initial amount of $N$ EPCs (say) are to be distributed. The first part of this experiment phase entails the implementation of a $k$-ary balanced search tree representing the $H L D$ for storing the location information of the EPCs. Every node of $H L D$ stores a list of structures for storing the timestamp and address of the PML server visited by the EPC of that node. The $H L D$ building phase takes as input the number of EPCs to distribute and their range.

The subsequent part of our experiment deals with populating the above database with simulated values. Before we begin simulating the distribution of EPCs, we define a population of PML servers and assign them addresses uniformly randomly. Using our discrete event driven framework, we simulate the distribution of the $N$ 
EPCs in this population and subsequently populate the fields of the search tree $H L D$, i.e., the address of the PML server where the corresponding EPC was distributed alongwith the simulated timestamp of distribution. For populating $H L D$, we have three events to simulate:

- The initiation of a new distribution at a node,

- The arrival of a sub-distribution at a node,

- The change of ownership in the distribution at a node, resulting in a new distribution.

The third event deals with the mapping of the current distributed EPC set $E$ to a new set $E_{\text {new }}$. We have assumed a range of 20-100 of the number of EPCs packaged or the number generated after depackaging an EPC. We generate the value uniformly randomly between these two limits.

An assumption that we make in our framework is that the EPCs to be distributed are contiguous in the EPC space. For the distribution phase, the number of EPCs to distribute is uniformly randomly chosen averaged around 1 million with a small variance. We define a distribution tree as a tree with each node representing a PML server and an edge representing an EPC distribution from the parent node to the child. The number of children PML servers at each node of the distribution tree is also generated uniformly randomly below a certain small threshold value which is a bound on the maximum children. At each node, the EPC set is further subdivided and propagated onto the children. The division of the current EPC set into subsets and distribution among the children PML servers is also uniform random. In our simulation we have assumed an upper bound of 20 on the depth of the distribution tree. This is a logical assumption, given the fact that there are not many sub-distributors in the hierarchy of a transaction which automatically puts a bound on the size of the smallest sub-distribution. Moreover, the probability of a redistribution from a node falls as the depth of distribution increases and if it is below a threshold then it is not redistributed. The third event is generated when the EPC is assumed to have reached its destination and there needs to be a mapping of the current set onto a different one. The processing of this event basically results in the generation of a new event of the first type which has the new set of EPCs for distribution. In our simulation the decision to map the old set onto the new set depends on a predefined probability value. We simulated 100 distributions and take the average of these to calculate the average number of PML servers that the EPCs got distributed to. After the construction of this simulated distribution tree, we populate the distribution information in the HLD. Thus each EPC in the HLD has a history chain indicating the distribution to different PML servers.

\section{B. Recall Phase}

In this phase, we are concerned with the recall of a random subset of the distributed EPCs. Without any loss of generality, the EPC set recalled is assumed to be contiguous in the EPC space. In order to compute the mean number of messages involved corresponding to each recall with a fixed EPC count, we average over 100 recalls, each corresponding to the same number of recalled EPCs but from different places in the EPC number space previously distributed. This gives us a stable value for the number of messages involved corresponding to the number of EPCs distributed and the number recalled.

This phase is straightforward once the HLD has been populated with all the location information with the time stamps. For each Recall, we construct the trie $T$ for the history of PML servers visited by the EPCs, by searching the location information list of each EPC in HLD. After $T$ is constructed, the total number of messages can be calculated by counting the number of nodes with nonempty EPC sets in $T$ alongwith their respective depth from the root (to account for the notifications backwards to the root) as explained in section III. If $R$ denotes the EPC set being recalled, then

$$
R=\bigcup_{i=1}^{z} R_{i}
$$

where $z$ is the total number of nodes where $R$ was distributed and $R_{i}$ is the subset distributed to the $i^{t h}$ node. Let $L_{i}$ represent the depth of the $i^{t h}$ node in $T$. Then the total number of messages required for the recall is equal to

$$
M=\sum_{i=1}^{z} L_{i}+z
$$

This is because after the recall messages are sent to each node $i$, they send acknowledgements or notification of the recall up the list of PML servers corresponding to the location history list of each $R_{i}$. Though this step is optional we have included it in the estimation of the number of messages generated. The first term denotes the total number of acknowledgements and the second term is the $z$ recall messages to the $z$ nodes. Figure 7 shows the simulation dynamics against time of the total number of PML servers affected by the EPC distribution out of a total of 100 PML servers. The plots are for various probabilities of infection acquiring. In the simulation, this value is calculated by the total amount of EPCs being distributed. The plots follow closely the dynamics of the total number infected as analytically shown in fig 6 .

Figure 8 depicts the average number of recall messages per EPC against the actual number recalled. This is plotted for various values of EPCs actually distributed apriori. The plot shows that after the initial values, the number of messages needed to perform the recall drops sharply as the number of recalled EPCs increases. Very small differences exist between the average number of messages 


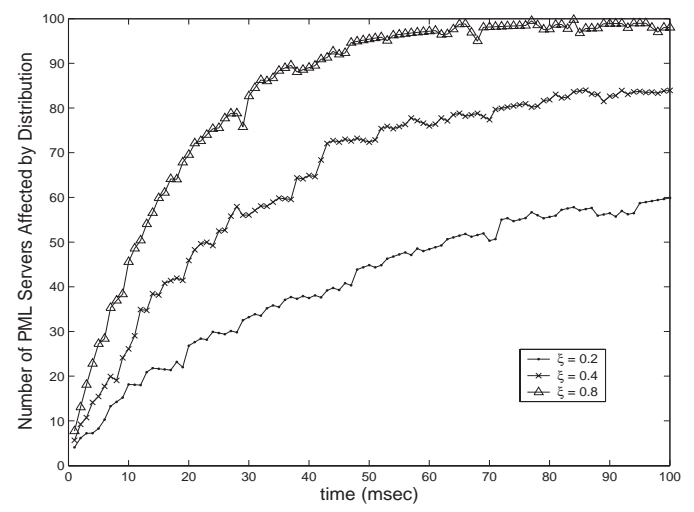

Fig. 7. Dynamics of distribution spread with time

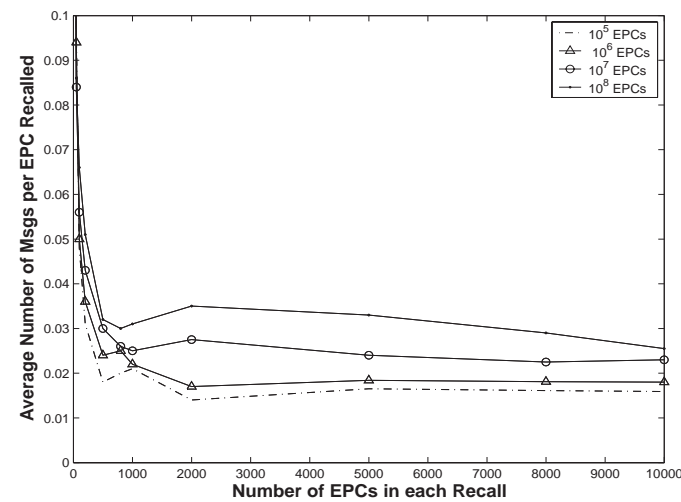

Fig. 8. Average Number of Messages w.r.t Recall Size

corresponding to different values of EPCs distributed. The curves are plotted with an order of magnitude difference in the number of EPCs distributed.

In Figure 9, the dynamics of the number of messages per EPC is shown with the number of EPCs actually distributed. The plot is shown till the 10 million mark. The number of messages per EPC increase with the number of distributed EPCs but they level out for larger values.

It is clear from both the plots, that the number of recall messages per EPC becomes very small for the large number of EPCs distributed which is a definite indicator to the efficacy of the adopted scheme for Object Recalls.

\section{Conclusions}

In this paper, we have proposed an architecture for automatic tracking of RFID tagged physical objects. We have further demonstrated a new scenario for Object Recall and proposed algorithms to deal with them based on our architecture. Using concepts of Scale Free Networks

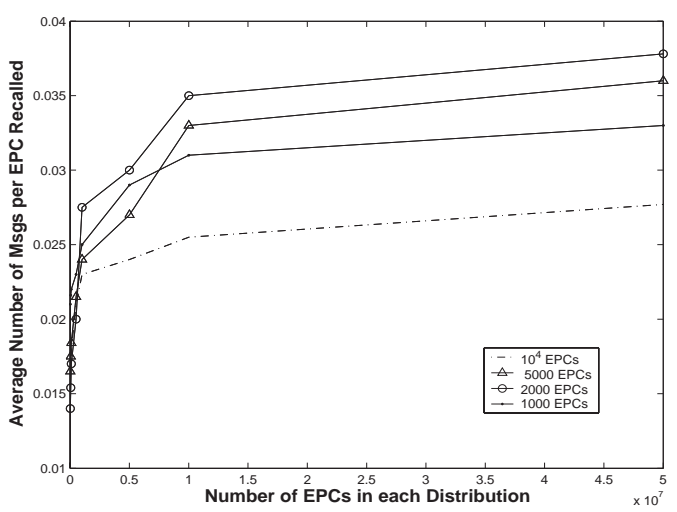

Fig. 9. Average Number of Messages w.r.t Distribution Size

and Epidemiology we have mathematically modelled the distribution process to estimate the messaging cost of the Recall Process. Our simulation results indicate that the average number of generated messages for each recall is indeed very low and the use of this recall handling framework can provide high performance benefits. Further details of this work is presented in [1].

\section{References}

[1] P. De, K. Basu, and S. K. Das, "An Ubiquitous Architectural Framework and Protocol for Object Tracking using RFID Tags", In Proceedings of the First International Conference on Mobile and Ubiquitous Systems(Mobiquitous), 2004.

[2] J.A. Mills, "Large scale interoperability and distributed transaction processing", In Proceedings of the Second International Conference on Systems Integration, 1992. ICSI 1992.

[3] R. Chang and K. Chen, "Dynamic mobility tracking for wireless personal communication networks", In Proceedings of IEEE 6th International Conference on Universal Personal Communications Record, 1997.

[4] P. Bhagwat, S. Tripathi and C. Perkins, "Network Layer Mobility: an Architecture and Survey", Technical Report, CS-TR-3570, University of Maryland (September 13, 1995).

[5] D. L. Brock, "The Electronic Product Code (EPC)- A Naming Scheme For Physical Objects", White Paper. MIT Auto-Id Center, January, 2001.

[6] The Savant - Version 0.1 (Alpha), Technical Manual. Oat Systems and MIT Auto-Id Center, February, 2002.

[7] D. L. Brock, T. P. Miline, Y. Y. Kang, and B. Lewis, "The Physical Markup Language", White Paper. MIT Auto-Id Center, June, 2001.

[8] H. Levy and Z. Naor, "An Active Tracking: Locating Mobile Users in Personal Communication Service Networks", ACM Journal on Wireless Networks, Vol. 5, No. 6, pp. 467-477, 1999.

[9] RFID Journal. http://www.rfidjournal.com/

[10] The Association for Automatic Identification and Data Capture Technologies. http://www.aimglobal.org/technologies/rfid/

[11] R. M. May and R. M. Anderson, Philos. Trans. R. Soc. London, Ser. B 321, 565 (1988).

[12] R. M. Anderson and R. M. May, Infectious Diseases of Human: Dynamics and Control (Oxford Univ. Press, Oxford, 1991).

[13] Handbook of Mathematical Functions, edited by A. Abramowitz and I. A. Stegun (Dover, New York, 1974).

[14] R. Albert et al, Nature (London)401, 130 (1999) 